\title{
Familial antithrombin III deficiency and Mycoplasma pneumoniae pneumonia
}

\author{
M D Creagh, I F Roberts, D J Clark, F E Preston
}

\begin{abstract}
A 10 year old girl presented with a massive femoral vein thrombosis associated with Mycoplasma pneumoniae pneumonia. Subsequently type I familial antithrombin II deficiency was diagnosed. It is suggested that prophylactic measures aimed at preventing thrombosis should be considered in acutely ill subjects with infection and familial thrombophilia.
\end{abstract}

Antithrombin III is a glycoprotein inhibitor of the activated coagulation factors thrombin (IIa), Xa, X11a, X1a and 1Xa. Moderate deficiency of antithrombin III is a potent cause of venous thromboembolic disease and may be acquired or inherited as an autosomal dominant disorder. It is estimated that within the general population the prevalence of symptomatic heterozygous antithrombin III deficiency is between 1 in $2000^{1}$ and 1 in $5000^{2}$ and that it is responsible for between $2-3 \%$ of episodes of venous thromboembolism in young adults. ${ }^{3}$

\section{Case report}

A previously well 10 year old girl presented with a two week history of intermittent fever, anorexia, and a non-productive cough, such that she had been bed-ridden for 12 days. Thirty six hours before admission she had noticed pain in the left groin, spreading to the thigh and calf. Medication had been oral erythromycin $0.5 \mathrm{~g}$ four times a day for four days.

Examination showed that she had a fever, a swollen left leg, and tenderness in the calf and groin. There were no focal signs in the respiratory system. A chest $x$ ray picture showed ill defined consolidation in the left middle lobe, compatible with $M$ pneumoniae pneumonia. The haemoglobin concentration was $12.3 \mathrm{~g} / \mathrm{l}$, neutrophils $18.6 \times 10^{9} / 1$, lymphocytes $3.1 \times 10^{9} / 1$ and platelets $400 \times$ $10^{9} / 1$. An ultrasound scan showed recent thrombi in the left femoral vein, which was confirmed by venogram.

Erythromycin $0.5 \mathrm{~g}$ four times a day was administered intravenously and the patient anticoagulated with an infusion of heparin, to which she initially proved resistant, and oral warfarin. M Pneumoniae infection was confirmed by an increase in the antibody titre from $1 / 240$ to $1 / 10000$. The patient's symptoms resolved and she was discharged on warfarin $1 \mathrm{mg}$ daily. Subsequent analysis of the patient's plasma for antithrombin III by radioimmunodiffusion and by functional chromogenic assay was consistent with type I antithrombin III deficiency, as were family studies of her 37 year old father and 45 year old uncle, neither of whom had a history of thrombosis (table).

Antithrombin III concentrations in patient and affected family members

\begin{tabular}{lll}
\hline & $\begin{array}{l}\text { Immunoreactive } \\
\text { antithrombin III }\end{array}$ & Functional \\
\hline Antithrombin III & & \\
Normal range & $0.79-1.11 \mathrm{IU} / \mathrm{ml}$ & $0.86-1.32 \mathrm{IU} / \mathrm{ml}$ \\
Patient & 0.65 & 0.70 \\
Father & 0.39 & 0.50 \\
Uncle & 0.59 & 0.63 \\
\hline
\end{tabular}

$\star$ By radioimmunodiffusion assay.

tBy chromogenic assay.

\section{Comment}

Since Egeberg first described an association between familial antithrombin III deficiency and thrombosis, ${ }^{4}$ many similar families have been described. There are two main types of familial antithrombin III deficiency: type I, in which there is reduced synthesis of a structurally normal molecule with parallel reduction of both immunoreactive and functional antithrombin III, and type II, due to a molecular variant, characterised by a normal immunoreactive antithrombin III with a low functional antithrombin III.

Those with the type I variant usually present between $20-40$ years of age; $5 \%$ of those affected will experience thrombosis in the first decade of life and $79 \%$ by the age of $40 . .^{5}$ Usually this is associated with immobility, trauma, surgery, pregnancy or use of combined oral contraceptives. In $30 \%$ there is no identifiable precipitating factor. In the case described there was a clear temporal association between the onset of femoral vein thrombosis and $M$ pneumoniae pneumonia. Although coagulation activation, manifest as disseminated intravascular coagulation, has been reported as a complication of $M$ pneumoniae infection its occurrence is nevertheless uncommon. The unusual circumstances prompted us to investigate the possibility of an underlying prothrombotic disorder; antithrombin III deficiency was diagnosed and its familial nature confirmed by detailed studies.

Another interesting observation shown here was that at the onset of thrombosis, before antithrombin III deficiency was recognised, it was difficult to achieve effective anticoagulation. This phenomenon has been described in other patients with antithrombin III 
deficiency $^{\circ}$ and reflects the importance of this serine protease inhibitor for the anticoagulant activity of heparin.

Our experience shows the importance of screening for an underlying disorder in the young with thrombosis and strongly suggests that prophylactic measures aimed at preventing thrombosis should be considered in acutely ill subjects with infection and familial thrombophilia.
1 Rosenberg RD. Actions and interactions of antithrombin and heparin. N Engl J Med 1975;292:146-51

2 Odegard OR, Abildgaard U. Antithrombin III: Critical review of assay methods. Significance of variations in health and disease. Haemostasis 1978;7:127-34.

3 Lechner K, Thaler E, Niessner H, Nowotny C, Portsch H. Antithrombin III mangel und thromboseneigung. Wien Klin Wochenschr 1977;89:215-22.

4 Egeberg $O$. Inherited antithrombin deficiency causing thrombophilia. Thromb Diath Haem 1965;13:516-30.

5 Hirsch J. Congenital antithrombin III deficiency. Am J Med 1989;87(suppl 3B):34-8.

6 Filip DJ, Eckstein JD, Veltkamp JJ. Hereditary antithrombin III deficiency and thromboembolic disease. $A m \mathrm{~J}$ Hematol 1976;2:343-9.

\title{
No evidence of HTLV-I infection in French patients with multiple sclerosis using the polymerase chain reaction
}

\author{
E Wattel, M Mariotti, J D Bignon, F Agis, E Gordien, J Y Muller, J Hors, J J Lefrère
}

Institut National de Transfusion Sanguine, Rue AlexandreCabanel, 75015, Paris, France

E Wattel

M Mariotti

J J Lefrere

Centre de Transfusion de Nantes, Allée Charles-Mirallié, Nantes, France J D Bignon J Y Muller

Centre de Transfusion, Guadeloupe, West

Indies

F Agis

E Gordien

Laboratoire de Génétique, Hôpital Saint-Louis, Paris, France

J Hors

Correspondence to: Dr Jean-Jacques Lefrère Accepted for publication 4 April 1991

\begin{abstract}
The polymerase chain reaction (PCR), using three primer pairs in the pol, tax, and $e n v$ regions of the HTLV-I genome, was unable to detect HTLV-I in the blood samples of 54 caucasian subjects with multiple sclerosis who were seronegative for HTLV-I/II. Seventeen HTLV-I/II seropositive (by ELISA and Western blot) subjects used as positive controls were positive with the three primer pairs. The PCR was negative in 47 healthy HTLV-I/II seronegative (by ELISA) subjects at low risk of HTLV-I infection used as negative controls.

These results suggest that there is no association between the occurrence of HTLV-I sequences and the development of multiple sclerosis.
\end{abstract}

The serological association between human T-lymphotropic virus type I (HTLV-I) infection and a chronic neurological disease such as the tropical spastic paraparesis prompted a search for this infection in other chronic neurological disorders such as multiple sclerosis (MS). The presence of specific antibodies ${ }^{12}$ and of HTLV-I sequences determined by in situ hybridisation $^{1}$ in patients with $M S$ has been disputed. ${ }^{3-4}$ Recently, Reddy ${ }^{5}$ and Greenberg, ${ }^{6}$ using gene amplification by the polymerase chain reaction (PCR), showed HTLV-I DNA sequences $^{5}$ or related retrovirus $^{6}$ in the peripheral blood mononuclear cells of patients with MS who were seronegative for HTLV-I.
As these results have not been confirmed by others, however, ${ }^{78}$ Reddy suggested that these discrepancies could have been due to the fact that only a subgroup of such cases could harbour HTLV-I sequences. ${ }^{5}$

Doubts therefore persist as to the possible relation between HTLV-I infection and MS. We decided to study a large number of French patients with MS to see if HTLV-I sequences could be detected using a PCR assay, to check if epidemiological differences could explain the discrepancies in previously published assays.

\section{Methods}

Fifty four caucasian adults with MS, as defined by Poser et $a l^{9}$, were studied. Mean age at onset of disease was 29.4 years; mean age at the time of the study was $42 \cdot 2$ years; 17 were male. All were seronegative by ELISA for antibodies to HTLV-I/II. None had a history of blood transfusions.

Seventeen HTLV-I/II seropositive (by ELISA) subjects living in an endemic region of HTLV-I/II infection (Guadeloupe, French West Indies) and showing typical patterns of HTLV-I/II infection on Western blotting were used as positive controls. Forty seven healthy HTLV-I/II seronegative (by ELISA) subjects (blood donors), living in a nonendemic area (Paris), and with no risk factors for HTLV-I/II infection were used as negative controls.

\section{DNA EXTRACTION}

DNA was prepared from $5 \mathrm{ml}$ of fresh or 